\title{
On near-critical and dynamical percolation in the tree case
}

\author{
Olle Häggström* Robin Pemantle ${ }^{\dagger}$ \\ Chalmers University of Technology and University of Wisconsin
}

June 18, 2018

\begin{abstract}
Consider independent bond percolation with retention probability $p$ on a spherically symmetric tree $\Gamma$. Write $\theta_{\Gamma}(p)$ for the probability that the root is in an infinite open cluster, and define the critical value $p_{c}=\inf \left\{p: \theta_{\Gamma}(p)>0\right\}$. If $\theta_{\Gamma}\left(p_{c}\right)=0$, then the root may still percolate in the corresponding dynamical percolation process at the critical value $p_{c}$, as demonstrated recently by Häggström, Peres and Steif. Here we relate this phenomenon to the near-critical behaviour of $\theta_{\Gamma}(p)$ by showing that the root percolates in the dynamical percolation process if and only if $\int_{p_{c}}^{1}\left(\theta_{\Gamma}(p)\right)^{-1} d p<\infty$. The "only if" direction extends to general trees, whereas the "if" direction fails in this generality.
\end{abstract}

\section{Introduction}

The main setup of this paper is that of independent bond percolation on an infinite locally finite connected graph $G=(V, E)$ with a distinguished vertex $\rho \in V$. Each edge $e \in E$ is independently assigned value 1 (open, on) with probability $p \in[0,1]$ and 0 (closed, off) with probability $1-p$; write $\mathbf{P}_{G, p}$ for the induced probability measure on $\{0,1\}^{E}$. Also write $\rho \leftrightarrow \infty$ for the event that $\rho$ is in an infinite open cluster, and define the percolation function $\theta_{G}(p)=\mathbf{P}_{G, p}[\rho \leftrightarrow \infty]$. Furthermore define the critical value $p_{c}=p_{c}(G)=\inf \left\{p: \theta_{G}(p)>0\right\}$. Let $\mathcal{C}$ denote the event that an infininte open cluster exists somewhere in $G$. It is well known that $P_{G, p}[\mathcal{C}]=0$ for $p<p_{c}$ and $P_{G, p}[\mathcal{C}]=1$ for $p>p_{c}$. At $p=p_{c}$, this probability can be either 0 or 1 , depending on whether $\theta_{G}\left(p_{c}\right)$ is 0 or strictly positive. This model has been studied extensively for several decades, mainly in the case where $G$ is the cubic lattice in $d \geq 2$ dimensions; see e.g. Grimmett [2].

We shall also consider a recent extension of the above setup, known as dynamical percolation. This is a time-dynamical variant which was introduced by Häggström, Peres and Steif [4]; see also Häggström [3] for a short survey. In this model, all edges $e \in E$ turn on and off according to independent stationary continuous time two-state Markov chains, turning on at rate $p$ and off at rate $1-p$ (that is, an edge in state 0 (resp. 1) waits for an amount of time whose distribution is exponential with mean $\frac{1}{p}$

\footnotetext{
${ }^{*}$ Research supported by a grant from the Swedish Natural Science Research Council.

${ }^{\dagger}$ Research supported in part by a Presidential Faculty Fellowship.
} 
(resp. $\frac{1}{1-p}$ ) before flipping to state 1 (resp. 0 )). We let $\Psi_{G, p}$ be a probability measure supporting such a $\{0,1\}^{E}$-valued Markov process. The stationary distribution for each edge puts probabilities $1-p$ and $p$ on states 0 and 1 , respectively. Hence, by the stationarity assumption, what we see at any fixed time $t$ is ordinary bond percolation with parameter $p$. Writing $\mathcal{C}_{t}$ for the event that an infinite open cluster exists at time $t$, we thus have (by Fubini's Theorem) that

$$
\Psi_{G, p}\left[\neg \mathcal{C}_{t} \text { occurs for a.e. } t\right]=1 \quad \text { if } \quad P_{p}[\mathcal{C}]=0
$$

and similarly

$$
\Psi_{G, p}\left[\mathcal{C}_{t} \text { occurs for a.e. } t\right]=1 \quad \text { if } \quad P_{p}[\mathcal{C}]=1 .
$$

[Here "a.e." is short for "(Lebesgue-)almost every". In other words, the left hand expression in (1) says that with $\Psi_{G, p}$-probability 1 , the set of times $t$ for which $\neg \mathcal{C}_{t}$ does not occur, has Lebesgue measure 0.] An obvious question to ask is whether the quantifier "a.e." can be strengthened to "every" in the above statements. For $p \neq p_{c}$, the answer is yes (see [4]), but (perhaps surprisingly) the answer is no for $p=p_{c}$ and certain choices of $G$. One of the main problems in dynamical percolation is the classification of graphs according to whether exceptional times exist at criticality.

Here we are interested in graphs for which $\theta_{G}\left(p_{c}\right)=0$ and in various notions of how "close" such a graph is to percolating at criticality. We can think of at least two reasonable ways of making "close" more precise.

1. The near-critical behaviour of $\theta_{G}(p)$. How rapidly does $\theta_{G}(p)$ take off from criticality? For instance, one can ask whether the derivative $\lim _{p} \searrow_{c} \frac{\theta_{G}(p)}{p-p_{c}}$ is finite or infinite. (Questions of this kind have been studied extensively in terms of so-called critical exponents, see e.g. Chapter 7 of Grimmett [2].)

2. Dynamical percolation. Will $\Psi_{G, p_{c}}$ assign positive probability to the existence of exceptional times at which an infinite open cluster exists?

Intuitively, one would expect that if $G$ is sufficiently close to percolating at criticality in one of these senses, then it should be close also in the other. The first result of this kind was obtained in [4], and says that if

$$
\limsup _{p \searrow p_{c}} \frac{\theta_{G}(p)}{p-p_{c}}<\infty
$$

then

$$
\Psi_{p_{c}}\left(\neg \mathcal{C}_{t} \text { occurs for every } t\right)=1 .
$$

Our goal here is to find sharper conditions of this kind in the case where $G$ is a tree, which with the currently available technology is virtually the only case that can be handled with some precision. An ultimate goal might be to find some "if and only if" criterion in terms of the near-critical behaviour of $\theta_{G}(p)$, determining whether there are percolating times for the critical dynamical percolation process. However, we shall see in Remark 2.4 below that a general criterion of this kind is impossible, at least if one insists that faster growth of $\theta_{G}(p)$ should make it easier to get percolating times in the dynamical percolation process. 
Let $\Gamma$ be an infinite locally finite tree with vertex set $V$, edge set $E$, and a distinguished vertex $\rho \in V$ called the root. For $v \in V$, write $|v|$ for the distance between $v$ and $\rho$. If $v$ and $w$ are nearest neighbours with $|w|=|v|+1$, then $w$ is called a child of $v$, and $v$ is called a parent of $w$. The vertex set $\{v \in V:|v|=n\}$ is called the $n$ :th level of $\Gamma$, and is denoted $\Gamma_{n}$. Further, $\left|\Gamma_{n}\right|$ denotes the number of vertices on the $n$ :th level. If two vertices at the same level always have the same number of children, then $\Gamma$ is called spherically symmetric.

Restricting to spherically symmetric trees, we do have a sharp criterion:

Theorem 1.1: Let $\Gamma$ be a spherically symmetric tree with $p_{c}(\Gamma)=p_{c} \in(0,1)$ and $\theta_{\Gamma}\left(p_{c}\right)=0$. Then

$$
\Psi_{\Gamma, p_{c}}(\rho \text { never percolates })=1
$$

if and only if

$$
\int_{p_{c}}^{1} \frac{1}{\theta_{\Gamma}(p)} d p=\infty
$$

Note that (4) is a strictly weaker condition than (2): An example of a spherically symmetric tree $\Gamma$ which satisfies (4) but not (2) can be obtained by letting $\left|\Gamma_{n}\right|$ be of the order $2^{n} \log n$ for each $n$. This can be shown using Lemma 2.1 below.

Only half of Theorem 1.1 can be extended to general trees:

Theorem 1.2: Let $\Gamma$ be an infinite locally finite tree with $p_{c}(\Gamma)=p_{c} \in(0,1)$ and $\theta_{\Gamma}\left(p_{c}\right)=0$. Then (4) implies (3). The converse is not true, i.e. there exists a choice of $\Gamma$ such that (3) holds and

$$
\int_{p_{c}}^{1} \frac{1}{\theta_{\Gamma}(p)} d p<\infty
$$

Our main motivation for this study was to try to shed some light on the important case where $G$ is the square lattice $\mathbf{Z}^{2}$ (more precisely, $V=\mathbf{Z}^{2}$ and $E$ consists of pairs of vertices at Euclidean distance 1 from each other). It is a classical result of Kesten [5] that $p_{c}=\frac{1}{2}$ and $\theta_{G}\left(p_{c}\right)=0$ for $G=\mathbf{Z}^{2}$. Moreover, Kesten and Zhang [6] showed that the condition (4) fails in this case. This means that if one could extend Theorem 1.1 to some class of graphs which includes $\mathbf{Z}^{2}$, then one would be able to conclude that for critical dynamical percolation on $\mathbf{Z}^{2}$ there would be exceptional times with infinite open clusters (this would contrast with the case of $\mathbf{Z}^{d}$ with $d$ sufficiently large; see [4]). One should not feel too discouraged by Theorem 1.2 in taking up this line of research, because the counterexample used to show that the "only if" direction of Theorem 1.1 does not hold in the generality of Theorem 1.2 is highly irregular and nonsymmetric. Theorem 1.1 thus provides some weak evidence that $\mathbf{Z}^{2}$ might have exceptional times at criticality. We want to stress, however, that we still do not think it is clear what the right conjecture should be. A different approach to the problem of critical dynamical percolation on $\mathbf{Z}^{2}$ is discussed by Benjamini, Kalai and Schramm [1].

\section{Proofs}

We first need some terminology on flows and electrical networks on trees; see e.g. Lyons and Peres [8] for a general introduction to this subject. An edge connecting two vertices 
$v, w \in V$ is denoted $\langle v, w\rangle$. A unit flow $\mathcal{F}$ on the tree $\Gamma$ is an assignment of non-negative numbers $\{\mathcal{F}(e)\}_{e \in E}$ to the edges of $\Gamma$, satisfying

(i) $\sum_{v} \mathcal{F}(\langle\rho, v\rangle)=1$, where the sum runs over all children of the root $\rho$, and

(ii) for each vertex $v$ (not equal to $\rho$ ) with parent $w$ and children $v_{1}, \ldots, v_{k}$, we have $\sum_{v_{i}} \mathcal{F}\left(\left\langle v, v_{i}\right\rangle\right)=\mathcal{F}(\langle w, v\rangle)$

This should be thought of as having "flow in equal to flow out" in all vertices except $\rho$ whose net flow out is 1 . An electrical network $\mathcal{C}$ on $\Gamma$ is simply an assignment of arbitrary positive numbers $\{\mathcal{C}(e)\}_{e \in E}$, called conductances, to the edges of $\Gamma$. The energy $W$ of a unit flow $\mathcal{F}$ in the network $\mathcal{C}$ is defined as

$$
W(\mathcal{F}, \mathcal{C})=\sum_{e \in E} \frac{(\mathcal{F}(e))^{2}}{\mathcal{C}(e)},
$$

and the effective conductance of the network $\mathcal{C}$ is defined as $[\inf \{W(\mathcal{F}, \mathcal{C})\}]^{-1}$ where the infimum runs over all unit flows $\mathcal{F}$ on $\Gamma$. This infimum is in fact a minimum, which moreover is attained at a unique unit flow whenever it is nonzero. Let $C(\Gamma, p)$ denote the effective conductance of the electrical network $N_{\Gamma, p}$ obtained by assigning each edge between $\Gamma_{n-1}$ and $\Gamma_{n}$ conductance $p^{n}$. Similarly, let $C^{*}(\Gamma, p)$ be the effective conductance the network $N_{\Gamma, p}^{*}$ in which edges between levels $n-1$ and $n$ are assigned conductance $n p^{n}$.

The keys to the proof of Theorem 1.1 are the following two results from the literature.

Lemma 2.1 (Lyons [7]): For each tree $\Gamma$ and each $p \in(0,1)$ we have

$$
\frac{C(\Gamma, p)}{1+C(\Gamma, p)} \leq \theta_{\Gamma}(p) \leq 2 \frac{C(\Gamma, p)}{1+C(\Gamma, p)}
$$

(See Marchal [9] for a recent sharpening of the upper bound in (6).)

Lemma 2.2 (Häggström, Peres and Steif [4]): For any tree $\Gamma$ with critical value $p_{c}$ we have that (3) holds if and only if

$$
C^{*}\left(\Gamma, p_{c}\right)=0 .
$$

Proof of Theorem 1.1: Since $\Gamma$ is spherically symmetric, we have

$$
C(\Gamma, \rho)=\left(\sum_{k=1}^{\infty} \frac{p^{-k}}{\left|\Gamma_{k}\right|}\right)^{-1}
$$

and

$$
C^{*}(\Gamma, \rho)=\left(\sum_{k=1}^{\infty} \frac{p^{-k}}{k\left|\Gamma_{k}\right|}\right)^{-1} .
$$

By Lemma 2.1, condition (4) can be rewritten as

$$
\int_{p_{c}}^{1} \frac{1+C(\Gamma, p)}{C(\Gamma, p)} d p=\infty
$$


which obviously is equivalent to

$$
\int_{p_{c}}^{1} \frac{1}{C(\Gamma, p)} d p=\infty
$$

By (7), this is the same as

$$
\int_{p_{c}}^{1} \sum_{k=1}^{\infty} \frac{p^{-k}}{\left|\Gamma_{k}\right|} d p=\infty
$$

which, in turn, is the same as

$$
\sum_{k=1}^{\infty} \frac{\int_{p_{c}}^{1} p^{-k} d p}{\left|\Gamma_{k}\right|}=\infty
$$

by Fubini's Theorem. The left hand side equals

$$
\frac{\ln \left(\frac{1}{p_{c}}\right)}{\left|\Gamma_{1}\right|}+\sum_{k=2}^{\infty} \frac{p_{c}^{-(k-1)}-1}{(k-1)\left|\Gamma_{k}\right|}
$$

so that (10) holds if and only if

$$
\sum_{k=1}^{\infty} \frac{p_{c}^{-k}}{k\left|\Gamma_{k}\right|}=\infty
$$

Now (8) and Lemma 2.2 complete the proof.

For the proof of Theorem 1.2, it is convenient to isolate the following lemma.

Lemma 2.3: For any $p, q \in(0,1)$, there exists a spherically symmetric tree $\Gamma$ with $p_{c}(\Gamma)=p$ and $\theta_{\Gamma}(p) \geq q$.

Proof: Fix $p$ and $q$. Let $\Gamma^{\prime}$ be a spherically symmetric tree with $\left|\Gamma_{k}^{\prime}\right|$ being bounded above and below by constants times $k^{2} p^{-k}$. Such a tree can be defined inductively by letting each vertex on level $k-1$ have exactly

$$
\min \left\{i \in\{1,2, \ldots\}: i\left|\Gamma_{k-1}^{\prime}\right| \geq k^{2} p^{-k}\right\}
$$

children. It is an easy application of Lemma 2.1 to show that $p_{c}\left(\Gamma^{\prime}\right)=p$ and $\theta_{\Gamma^{\prime}}(p)>0$. If $\theta_{\Gamma^{\prime}}(p) \geq q$ we are done by taking $\Gamma=\Gamma^{\prime}$. Otherwise we set

$$
j=\min \left\{i \in\{1,2, \ldots\}:\left(1-\theta_{\Gamma^{\prime}}(p)\right)^{j} \leq 1-q\right\} .
$$

and let $\Gamma$ consist of $j$ copies of $\Gamma^{\prime}$ sharing the same root $\rho$.

Proof of Theorem 1.2: We first show that (4) implies (3) by proving the equivalent statement that

$$
\Psi_{p_{c}}(\exists t \text { at which } \rho \text { percolates })>0
$$

implies (5). Suppose that (11) holds. We then have $C^{*}\left(\Gamma, p_{c}\right)>0$ by Lemma 2.2. Let $\mathcal{F}^{*}$ denote the minimal energy unit flow from $\rho$ to infinity in the electrical network $N_{\Gamma, p_{c}}^{*}$. Write $W^{*}$ for the energy of $\mathcal{F}^{*}$ in $N_{\Gamma, p_{c}}^{*}$, so that

$$
W^{*}=\frac{1}{C^{*}\left(\Gamma, p_{c}\right)}<\infty
$$


Decompose $W^{*}$ as

$$
W^{*}=\sum_{k=1}^{\infty} W^{*}(k)
$$

where $W^{*}(k)$ is the contribution to $W^{*}$ coming from edges connecting levels $k-1$ and $k$ in $\Gamma$. Also write $W_{p}$ for the energy of $\mathcal{F}^{*}$ in the network $N_{\Gamma, p}$, and decompose $W_{p}$ into

$$
W_{p}=\sum_{k=1}^{\infty} W_{p}(k)
$$

analogously to (13). We have

$$
\frac{1}{C(\Gamma, p)} \leq W_{p}
$$

and furthermore

$$
W_{p}(k)=k\left(\frac{p_{c}}{p}\right)^{k} W^{*}(k)
$$

Hence

$$
\begin{aligned}
\int_{p_{c}}^{1} \frac{1}{C(\Gamma, p)} d p & \leq \int_{p_{c}}^{1} \sum_{k=1}^{\infty} W_{p}(k) d p \\
& =\sum_{k=1}^{\infty} \int_{p_{c}}^{1} W_{p}(k) d p \\
& =\sum_{k=1}^{\infty} k W^{*}(k) \int_{p_{c}}^{1}\left(\frac{p_{c}}{p}\right)^{k} d p \\
& =p_{c} \sum_{k=1}^{\infty} \frac{k\left(1-p_{c}^{k-1}\right)}{k-1} W^{*}(k) \\
& <\infty
\end{aligned}
$$

where the last step is due to (13) and (12). The desired conclusion (5) follows using Lemma 2.1.

It remains to give an example of a tree $\Gamma$ for which (3) and (5) both hold. For each $p \in(1 / 2,1)$, let $\Gamma^{(p)}$ denote some tree with critical value $p_{c}\left(\Gamma^{(p)}\right)=p$ and $\theta_{\Gamma^{(p)}}(p) \geq 1 / 2$; such trees exist by Lemma 2.3. The construction of $\Gamma$ is as follows. Start with a single infinite branch $\left(\rho, v_{1}, v_{2}, \ldots\right)$ and attatch to each vertex $v_{i}$ a copy of $\Gamma^{\left(p_{i}\right)}$, where the sequence $\left\{p_{i}\right\}_{i \geq 1}$ is decreasing with $p_{i}>1 / 2$ for each $i$ and $\lim _{i \rightarrow \infty} p_{i}=1 / 2$. We get $p_{c}(\Gamma)=1 / 2$. Furthermore, $\theta_{\Gamma}\left(p_{i}\right) \geq 2^{-(i+1)}$, so that we can make $\theta_{\Gamma}(p)$ take off from criticality arbitrarily fast by letting the $p_{i}$ 's tend to $1 / 2$ sufficiently fast. In particular, we can make (5) hold by e.g. taking $p_{i}=1 / 2+3^{-i}$. On the other hand, note that percolation on $\Gamma^{\left(p_{i}\right)}$ is subcritical at $p=1 / 2$ for each $i$, whence

$$
\Psi_{\Gamma^{\left(p_{i}\right)}, 1 / 2}\left[\forall t \neg \mathcal{C}_{t}\right]=1 .
$$

Since the existence of an infinite open cluster in $\Gamma$ implies the existence of an infinite cluster either in the branch $\left(\rho, v_{1}, v_{2}, \ldots\right)$ or in one of the subtrees $\Gamma^{\left(p_{i}\right)}$, we get

$$
\Psi_{\Gamma, 1 / 2}\left[\forall t \neg \mathcal{C}_{t}\right]=1,
$$


so the proof is complete.

Remark 2.4: The construction at the end of the proof of Theorem 1.2 shows that we can find two trees $\Gamma$ and $\Gamma^{\prime}$ with the properties that

(i) $p_{c}(\Gamma)=p_{c}\left(\Gamma^{\prime}\right)=1 / 2$,

(ii) $\theta_{\Gamma}(1 / 2)=\theta_{\Gamma^{\prime}}(1 / 2)=0$,

(iii) the critical dynamical percolation process has times with infinite clusters for $\Gamma$ but not for $\Gamma^{\prime}$, and

(iv) there exists a $p^{*}>1 / 2$ such that $\theta_{\Gamma}(p)<\theta_{\Gamma^{\prime}}(p)$ for all $p \in\left(1 / 2, p^{*}\right)$.

Indeed, take $\Gamma$ to be spherically symmetric with $\left|\Gamma_{n}\right|$ of the order $n 2^{n}$, and then build up $\Gamma^{\prime}$ as in the above construction sending $p_{i}$ to $1 / 2$ fast enough so that (iv) holds. In words, (i)-(iv) tell us that $\Gamma^{\prime}$ is closer to percolating at criticality than $\Gamma$ in the sense of how fast $\theta(p)$ takes off from criticality, whereas $\Gamma$ is closer to percolating in the sense of dynamical percolation.

\section{References}

[1] Benjamini, I., Kalai, G. and Schramm, O. (1998) Noise sensitivity of boolean functions and applications to percolation, preprint.

[2] Grimmett, G. (1989) Percolation, Springer, New York.

[3] Häggström, O. (1998) Dynamical percolation: early results and open problems, Microsurveys in Discrete Probability (D. Aldous and J. Propp, eds), DIMACS Series on Discrete Mathematics and Theoretical Computer Science 41, pp 59-74, American Mathematical Society, Providence, RI.

[4] Häggström, O., Peres, Y. and Steif, J.E. (1997) Dynamical percolation, Ann. Inst. H. Poincaré Probab. Statist. 33, 497-528.

[5] Kesten, H. (1980) The critical probability of bond percolation on the square lattice equals $\frac{1}{2}$, Commun. Math. Phys. 74, 41-59.

[6] Kesten, H. and Zhang, Y. (1987) Strict inequalites for some critical exponents in 2Dpercolation, J. Statist. Phys. 46, 1031-1055.

[7] Lyons, R. (1992) Random walks, capacity, and percolation on trees, Ann. Probab. 20, 2043-2088.

[8] Lyons, R. and Peres, Y. (1997) Probability on Trees and Networks, Cambridge University Press, in preparation. Current version available at http://php.indiana.edu/ ${ }^{\sim}$ rdlyons/

[9] Marchal, P. (1998) The best bounds in a theorem of Russell Lyons, Electr. Commun. Probab. 3, 91-94. 